\title{
Treatment Initiation Following Positive Depression Screens in Primary Care: a Propensity Score-Weighted Analysis of Integrated Mental Health Services
}

\author{
Brittany L. Cornwell, MPH ${ }^{1,2}$, Benjamin R. Szymanski, $P h D, M P H^{7}$, \\ Kipling M. Bohnert, $P h D^{1,3,4}$, and John F. McCarthy, PhD, MPH $H^{1,3,4}$
}

\begin{abstract}
'Serious Mental Illness Treatment Resource and Evaluation Center, Office of Mental Health and Suicide Prevention, Department of Veterans Affairs, Ann Arbor, MI, USA; ${ }^{2}$, Grand Rapids, USA; ${ }^{3}$ Department of Psychiatry, University of Michigan, Ann Arbor, MI, USA; ${ }^{4}$ Center for Clinical Management Research, Department of Veterans Affairs, Ann Arbor, MI, USA.
\end{abstract}

J Gen Intern Med 36(2):561-3

DOI: $10.1007 / \mathrm{s} 11606-020-05694-5$

(c) Society of General Internal Medicine (This is a U.S. government work and not under copyright protection in the U.S.; foreign copyright protection may apply) 2020

I n 2007, the Veterans Health Administration (VHA) began national implementation of Primary Care-Mental Health Integration (PCMHI) services. A major goal was to enhance mental health access and address unmet treatment needs. Integrated care services have been shown to improve depression identification ${ }^{1}$ and enhance engagement in specialty mental health treatment. ${ }^{2}$

Szymanski et al. examined a sample of VHA users in fiscal year (FY) 2010 and documented positive associations between receipt of PCMHI services on the day of a positive depression screen and initiation of depression treatment within 12 weeks. ${ }^{3}$ The authors noted as a study limitation that individuals who had received same-day PCMHI services may have had unmeasured differences in symptom severity or willingness to initiate depression treatment, as compared with other study patients. ${ }^{3}$ Also, in the years since 2010, VHA implementation of PCMHI services has expanded substantially. The present analysis reevaluates the influence of same-day PCMHI services on initiation of depression treatment, for a more recent period and adjusting for patient propensity to have received PCMHI and/ or Specialty Mental Health (SMH) clinic services on the day of the initial positive depression screen in primary care (PC).

\section{METHODS}

For FY2017 data, we applied methods from the FY2010 study, ${ }^{3}$ adding use of propensity score weights to adjust for patient likelihood of receiving same-day PCMHI and/or SMH

Received January 24, 2020

Accepted January 31, 2020

Published online June 3, 2020 services. ${ }^{4,5}$ Generalized boosted models ${ }^{4,5}$ were used to create the weights, with location of same-day services as the outcome. Separate propensity score-weighted logistic regressions assessed initiation within 12 weeks of antidepressant pharmacotherapy, psychotherapy, and either treatment. ${ }^{4}$

Analyses used SAS (version 9.3) and R software (version 3.4.2). The study was conducted as part of ongoing operations in the VHA Office of Mental Health and Suicide Prevention.

\section{RESULTS}

Table 1 presents sample characteristics, by type of services received. Compared with patients receiving same-day PCMHI, the "PC only" patients were older $(38.4 \%$ vs. $23.1 \%$ were $65+$ years old); less likely to be Hispanic (7.7\% vs. $8.9 \%)$; more likely to be male ( $87.8 \%$ vs. $85.7 \%)$, married $(49.2 \%$ vs. $45.8 \%)$, with a prior mental health diagnosis $(16.7 \%$ vs. $13.3 \%)$ and prior VHA outpatient use $(67.1 \%$ vs. 64.6\%); and had lower baseline PHQ-2 (Patient Health Questionnaire) scores (4.64 vs. 4.83).

Table 2 presents the multivariable logistic regression analyses with propensity score adjustment. Compared with patients seen only in PC, patients receiving same-day PCMHI had 9.36 (95\% CI, 8.75-10.00) times greater odds of receiving psychotherapy, 2.46 (95\% CI, 2.30-2.62) times greater odds of receiving an antidepressant prescription fill, and 7.73 (95\% CI, 7.20-8.30) times greater odds of receiving either treatment, all within 12 weeks of the initial screen.

\section{DISCUSSION}

Patients receiving same-day PCMHI have greater odds of initiating timely depression treatment. This was observed even when adjusting for observed likelihood of receiving these services, per available measures of morbidity and utilization. Findings support prioritization of efforts to enhance same-day PCMHI access, including support for collaboration between PC and PCMHI providers, for patients with positive depression screens. 


\begin{tabular}{|c|c|c|c|c|c|}
\hline & \multirow[b]{2}{*}{$\begin{array}{c}\text { Overall } \\
\begin{array}{c}(\mathrm{N}=92,535) \\
\mathrm{N}(\%)\end{array}\end{array}$} & \multicolumn{4}{|c|}{ Same-Day Mental Health Care Receipt } \\
\hline & & $\begin{array}{l}\text { None, Primary } \\
\text { Care Only } \\
(\mathrm{N}=76,564) \\
\mathrm{N}(\%)\end{array}$ & $\begin{array}{c}\text { PCMHI } \\
(N=6,748) \\
N(\%) \\
\end{array}$ & $\begin{array}{l}\text { PCMHI and } \\
\text { SMH } \\
(\mathrm{N}=5,625) \\
\mathrm{N}(\%) \\
\end{array}$ & $\begin{array}{c}\text { SMH } \\
(\mathrm{N}=3,598) \\
\mathrm{N}(\%) \\
\end{array}$ \\
\hline Age & & & $* *$ & $* *$ & $* *$, \\
\hline$\leq 44$ & $25,037(27.1)$ & $18,539(24.2)$ & $2,732(40.5)$ & $2,327(41.4)$ & $1,439(40.0)$ \\
\hline $45-64$ & 34,627 (37.4) & $28,617(37.4)$ & 2,459 (36.4) & $2,065(36.7)$ & $1,486(41.3)$ \\
\hline$\geq 65$ & $32,871(35.5)$ & $29,408(38.4)$ & $1,557(23.1)$ & $1,233(21.9)$ & $673(18.7)$ \\
\hline \multicolumn{6}{|l|}{ Sex } \\
\hline Female & $11,599(12.5)$ & $9,366(12.2)$ & $966(14.3)^{* *}$ & $751(13.4)^{*}$ & $516(14.3)^{* *}$ \\
\hline Race & & & $* *$ & & $* *, \notin$ \\
\hline White & $59,211(64.0)$ & $49,235(64.3)$ & $4,330(64.2)$ & $3,571(63.5)$ & $2,075(57.7)$ \\
\hline African American & $23,320(25.2)$ & 19,044 (24.9) & 1660 (24.6) & $1,444(25.7)$ & $1,172(32.6)$ \\
\hline Other & $4,616(5.0)$ & 3,757 (4.9) & $405(6.0)$ & $281(5.0)$ & $173(4.8)$ \\
\hline Unknown & $5,388(5.8)$ & $4,528(5.9)$ & $353(5.2)$ & 329 (5.9) & $178(5.0)$ \\
\hline Hispanic & $7,369(8.0)$ & $5,927(7.7)$ & $598(8.9)^{*}$ & $565(10.0)^{* *}$ & $279(7.8)$ \\
\hline Marital status & & & $* *$ & $* *$ & $* *, \notin$ \\
\hline Married & $44,568(48.2)$ & $37,688(49.2)$ & $3,092(45.8)$ & $2,517(44.8)$ & 1,271 (35.3) \\
\hline Not Married & 31,685 (34.2) & $26,221(34.3)$ & $2,240(33.2)$ & $1,856(33.0)$ & $1,368(38.0)$ \\
\hline Never Married & 14,969 (16.2) & $11,613(15.2)$ & $1,312(19.5)$ & $1,142(20.3)$ & $902(25.1)$ \\
\hline Unknown & $1,313(1.4)$ & $1,042(1.4)$ & $104(1.5)$ & $110(2.0)$ & $57(1.6)$ \\
\hline \multicolumn{6}{|l|}{ Service connected } \\
\hline $70+\%$ & $36,386(39.3)$ & 30,149 (39.4) & $2,600(38.5)$ & $2,101(37.4)^{*}$ & $1,536(42.7)^{* *}, €$ \\
\hline \multicolumn{6}{|l|}{$\begin{array}{l}\text { Mental Health } \\
\text { diagnoses in prior } \\
\text { year }\end{array}$} \\
\hline Anxiety & $3,909(4.2)$ & $3,133(4.1)$ & $254(3.8)$ & $250(4.4)$ & $272(7.6)^{* *}, €$ \\
\hline PTSD & 8,915 (9.6) & $7,368(9.6)$ & $466(6.9)^{* *}$ & $388(6.9)^{* *}$ & $693(19.3)^{* *}, €$ \\
\hline SMI & $1,875(2.0)$ & $1,393(1.8)$ & $82(1.2)^{* *}$ & $89(1.6)$ & $311(8.6)^{* *}, \pm$ \\
\hline SUD & $2,529(2.7)$ & $1,887(2.5)$ & $130(1.9)^{*}$ & $158(2.8)$ & $354(9.8)^{* *}, \mathrm{t}$ \\
\hline Other & $2,780(3.0)$ & 2,185 (2.9) & $192(2.9)$ & $177(3.2)$ & $226(6.3)^{* *}, €$ \\
\hline Any of the above & $15,893(17.2)$ & 12,805 (16.7) & $898(13.3)^{* *}$ & $856(15.2)^{*}$ & $1,334(37.1)^{* *}, \pm$ \\
\hline $\begin{array}{l}\text { Number of PC } \\
\text { encounters in prior } \\
12 \text { months }^{\wedge}\end{array}$ & $3.04(4.31)$ & $3.21(4.40)$ & $2.03(3.42)^{* *}$ & $2.13(3.56)^{* *}$ & $2.71(4.56)^{* *}, \notin$ \\
\hline $\begin{array}{l}\text { No prior VHA } \\
\text { outpatient use }\end{array}$ & $31,016(33.5)$ & $25,204(32.9)$ & $2,386(35.4)^{* *}$ & $\begin{array}{r}2,062 \\
(36.7)^{* *}\end{array}$ & $1,364(37.9)^{* *}, \ddagger$ \\
\hline PHQ-2 Score^ & $4.68(1.23)$ & $4.64(1.23)$ & $4.83(1.19)^{* *}$ & $4.89(1.19)^{* *}$ & $4.86(1.21)^{* *}$ \\
\hline
\end{tabular}

$\wedge$ Presented as mean (SD)

$*_{p}<0.05$ compared to PC only

$\ddagger p<0.05$ compared to PCMHI only

$\notin p<0.001$ compared to PCMHI only

$* * p<0.001$ compared to PC only

Study findings are consistent with those reported for FY2010, ${ }^{3}$ with FY2017 sensitivity analyses without propensity score weights, and with other assessments of the impact of same-day integrated care services. A national VHA study examining patients with a positive PC-PTSD screen found that same-day PCMHI services were associated with greater PTSD treatment initiation compared to patients receiving only $\mathrm{PC}$ services. ${ }^{6}$ This work extends previous findings demonstrating the effectiveness of PCMHI on diagnosis and treatment of common mental health conditions by providing propensity scoreadjusted evidence of the value of same-day PCMHI for depression treatment facilitation. 
Table 2 Adjusted Odds Ratios for Treatment Initiation Within 12 Weeks of Initial Positive Depression Screen*

\begin{tabular}{|c|c|c|c|c|c|c|c|c|c|}
\hline & \multicolumn{3}{|c|}{ Psychotherapy } & \multicolumn{3}{|c|}{ Antidepressant } & \multicolumn{3}{|c|}{ Either treatment } \\
\hline & OR & $\begin{array}{l}95 \% \mathrm{CI} \\
\mathrm{LL}\end{array}$ & $\begin{array}{l}95 \% \mathrm{CI} \\
\text { UL }\end{array}$ & OR & $\begin{array}{l}95 \% \mathrm{CI} \\
\text { LL }\end{array}$ & $\begin{array}{l}95 \% \mathrm{CI} \\
\text { UL }\end{array}$ & OR & $\begin{array}{l}95 \% \mathrm{CI} \\
\text { LL }\end{array}$ & $\begin{array}{l}\text { 95\% CI } \\
\text { UL }\end{array}$ \\
\hline \multicolumn{10}{|l|}{ Location } \\
\hline PC only & Ref & & & Ref & & & Ref & & \\
\hline PCMHI & 9.36 & 8.75 & 10.00 & 2.46 & 2.30 & 2.62 & 7.73 & 7.20 & 8.30 \\
\hline PCMHI and SMH & 11.77 & 10.90 & 12.71 & 2.78 & 2.59 & 2.98 & 10.36 & 9.52 & 11.28 \\
\hline $\mathrm{SMH}$ & 4.48 & 4.08 & 4.91 & 2.21 & 2.01 & 2.43 & 3.92 & 3.57 & 4.30 \\
\hline \multicolumn{10}{|l|}{ Age } \\
\hline$\leq 44$ & Ref & & & Ref & & & Ref & & \\
\hline$\overline{4} 5-64$ & 0.81 & 0.75 & 0.88 & 0.69 & 0.64 & 0.74 & 0.73 & 0.68 & 0.79 \\
\hline$\geq 65$ & 0.65 & 0.59 & 0.71 & 0.46 & 0.41 & 0.50 & 0.51 & 0.46 & 0.56 \\
\hline \multicolumn{10}{|l|}{ Sex } \\
\hline Female & Ref & & & Ref & & & Ref & & \\
\hline Male & 0.92 & 0.83 & 1.01 & 0.83 & 0.76 & 0.91 & 0.82 & 0.75 & 0.91 \\
\hline \multicolumn{10}{|l|}{ Race } \\
\hline White & Ref & & & Ref & & & Ref & & \\
\hline African American & 1.00 & 0.92 & 1.09 & 0.87 & 0.81 & 0.95 & 0.91 & 0.84 & 1.00 \\
\hline Other & 1.06 & 0.90 & 1.24 & 0.99 & 0.86 & 1.15 & 0.99 & 0.84 & 1.17 \\
\hline Unknown & 0.82 & 0.70 & 0.96 & 0.91 & 0.78 & 1.07 & 0.89 & 0.77 & 1.04 \\
\hline Hispanic & 1.08 & 0.94 & 1.23 & 0.87 & 0.77 & 0.98 & 0.99 & 0.87 & 1.13 \\
\hline \multicolumn{10}{|l|}{ Marital status } \\
\hline Married & Ref & & & Ref & & & Ref & & \\
\hline Not married & 0.91 & 0.84 & 0.98 & 0.84 & 0.78 & 0.90 & 0.87 & 0.80 & 0.94 \\
\hline Never married & 0.92 & 0.84 & 1.01 & 0.77 & 0.70 & 0.84 & 0.86 & 0.78 & 0.94 \\
\hline Unknown & 0.98 & 0.76 & 1.26 & 1.05 & 0.84 & 1.32 & 1.06 & 0.82 & 1.37 \\
\hline $\begin{array}{l}\text { Service connected } \\
\quad 70+\%\end{array}$ & 1.18 & 1.10 & 1.28 & 1.14 & 1.06 & 1.22 & 1.16 & 1.08 & 1.25 \\
\hline $\begin{array}{l}\text { Number of PC encounters in prior } \\
12 \text { months }\end{array}$ & 0.97 & 0.96 & 0.98 & 0.96 & 0.95 & 0.97 & 0.97 & 0.96 & 0.98 \\
\hline No prior VHA outpatient use & 1.24 & 1.15 & 1.33 & 0.93 & 0.87 & 1.00 & 1.15 & 1.07 & 1.24 \\
\hline PHQ-2 score & 1.05 & 1.02 & 1.08 & 1.12 & 1.09 & 1.15 & 1.09 & 1.06 & 1.12 \\
\hline \multicolumn{10}{|c|}{ Mental health diagnoses in prior year } \\
\hline Anxiety & 1.14 & 0.97 & 1.34 & 1.10 & 0.94 & 1.27 & 1.17 & 1.00 & 1.37 \\
\hline PTSD & 1.18 & 1.05 & 1.32 & 0.83 & 0.74 & 0.93 & 1.13 & 1.01 & 1.27 \\
\hline SMI & 1.01 & 0.80 & 1.27 & 0.37 & 0.28 & 0.49 & 0.78 & 0.62 & 0.97 \\
\hline SUD & 1.32 & 1.11 & 1.56 & 0.81 & 0.68 & 0.97 & 1.13 & 0.95 & 1.34 \\
\hline Other & 1.23 & 1.03 & 1.47 & 0.72 & 0.61 & 0.85 & 1.06 & 0.89 & 1.26 \\
\hline
\end{tabular}

OR odds ratio, CI confidence interval, PC primary care, PCMHI Primary Care-Mental Health Integration, SMH Specialty Mental Health, PHQ Patient Health Questionnaire

*Logistic regression models were weighted to account for likelihood of receiving same-day PCMHI and/or SMH services. The facility where patients received their initial positive depression screen was included as a covariate in the models, ORs not shown

A limitation of this study is that patients may have unmeasured differences in treatment needs. Although all screened positive for depression, screening is not equivalent to diagnosis and only $23 \%$ of patients received a same-day depression diagnosis. Nonetheless, in a sensitivity analysis limited to patients who received a same-day depression diagnosis, the odds of initiating either treatment remained significantly higher among patients receiving same-day PCMHI.

After more than a decade of VA PCMHI service implementation, and 7 years after the initial assessment, this study documents that same-day PCMHI services continue to have positive associations with treatment engagement among patients with positive depression screens. Findings support ongoing efforts to ensure the availability of PCMHI services, including same-day services access.

Acknowledgments: This study was conducted as part of the Veterans Health Administration (VHA) Primary Care Mental-Health Integration National Evaluation. We thank VHA program, operations, and policy leaders regarding this report.

Corresponding Author: Brittany L. Cornwell, MPH; , Grand Rapids, USA (e-mail: Brittany.Cornwell@va.gov).

\section{Compliance with Ethical Standards:}

Conflict of Interest: The authors declare that they do not have a conflict of interest.

\section{REFERENCES}

1. Pomerantz AS, Cole BH, Watts BV, Weeks WB: Improving efficiency and access to mental health care: Combining integrated care and advanced access. Gen Hosp Psychiatry 30:546-551, 2008

2. Wray LO, Szymanski BR, Kearney LK, et al: Implementation of primary care-mental health integration services in the Veterans Health Administration: Program activity and associations with engagement in specialty mental health services. J Clin Psychol Med Settings 19:105-116, 2012

3. Szymanski BR, Bohnert KM, Zivin $\mathbf{K}$, et al: Integrated care: treatment initiation following positive depression screens. J Gen Intern Med 28:346352, 2013

4. McCaffrey DF, Griffin BA, Almirall D, et al: A tutorial on propensity score estimation for multiple treatments using generalized boosted models. Stat Med 32(19):3388-3414, 2013

5. Ridgeway G, McCaffrey D, Morral A, et al. Tooklit for weighting and analysis of nonequivalent groups: a tutorial for the twang package. RAND Corporation. June 16, 2016. Available at www.rand.org. Accessed Sep 21, 2018

6. Bohnert KM, Sripada RK, Mach J, et al: Same-day integrated mental health care and PTSD diagnosis and treatment among VHA primary care patients with positive PTSD screens. Psychiatr Serv 67(1):94-100, 2015

Publisher's Note Springer Nature remains neutral with regard to jurisdictional claims in published maps and institutional affiliations. 